\title{
Evolution of multiple black hole nuclei in merged galaxies
}

\author{
Mauri Valtonen, Seppo Mikkola and Pekka Heinämäki \\ Tuorla Observatory and Physics Department, University of Turku, \\ 21500 Piikkiö, Finland
}

\begin{abstract}
The evolution of three and four black hole clusters which arise in mergers of small galaxy groups have been simulated. Typically, the chaotic dynamics of these multiple black hole systems leads to a decay via the slingshot process. Orbits of 100,000 initial configurations have been calculated and the final configurations have been classified in seven different categories. Examples of the orbits in each category are illustrated.
\end{abstract}

\section{Introduction}

Calculations of the dynamical evolution of black holes within merging galaxies in small groups were carried out using the Newtonian force law with an additional gravitational radiation term by Peters and Mathews (1963). Calculations started at the point where two binary black hole systems have formed in the nucleus of the merger. Initial conditions of the black holes were chosen to make the starting configuration as realistic as possible in the merger situation. The calculations include 10,000 cases in 10 different sets of randomly generated three and four black hole systems. For a more detailed description, see Valtonen et al. (1994).

Integrations of the orbits were carried out until either only two bodies remain in the galaxy or 100 crossing times of the initial system were completed, or the 5000 time step limit was reached. A black hole was identified as an escaper if the velocity of the body relative to the centre of mass exceeded the escape velocity of the host galaxy (taken to be about $2000 \mathrm{~km} \mathrm{~s}^{-1}$ ) while the body was at large distance from the center of mass and moving away from it. At that point the final states of the systems were classified in seven categories. Examples of the orbits leading to each of the seven final categories are illustrated in Fig. 1. The categories are:

Case 1. Two bodies left in the galaxy.

Case 2. Two bodies left in the galaxy; single body or binary escapes.

Case 3. Two bodies left in the galaxy; two bodies escape.

Case 4. One body left in the galaxy; single body or binary escapes.

Case 5. One body left in the galaxy; two bodies escape.

Case 6. Nothing left in the galaxy; two single bodies or binaries escape.

Case 7. Nothing left in the galaxy; three single bodies escape. 


\section{Case1 Case2}

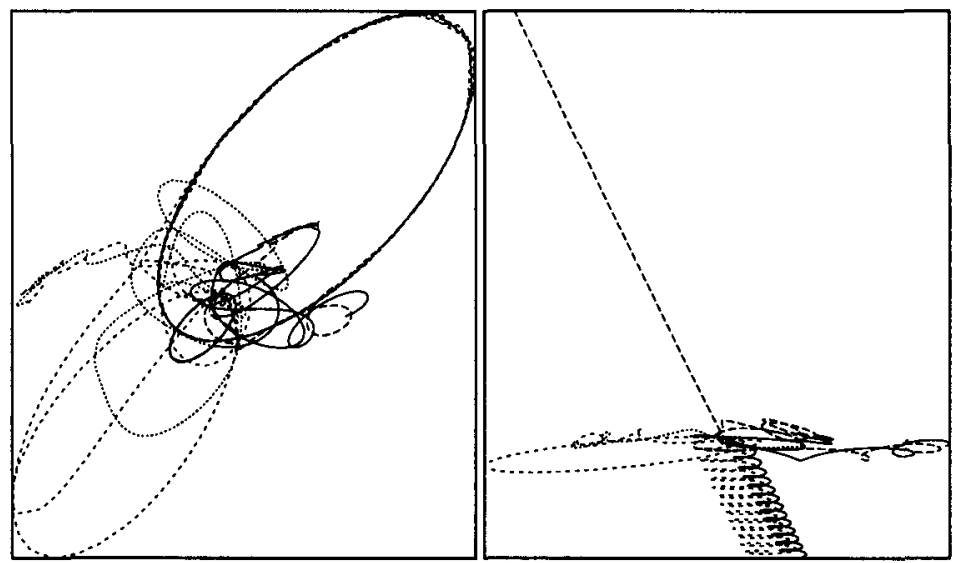

Case3

Case4

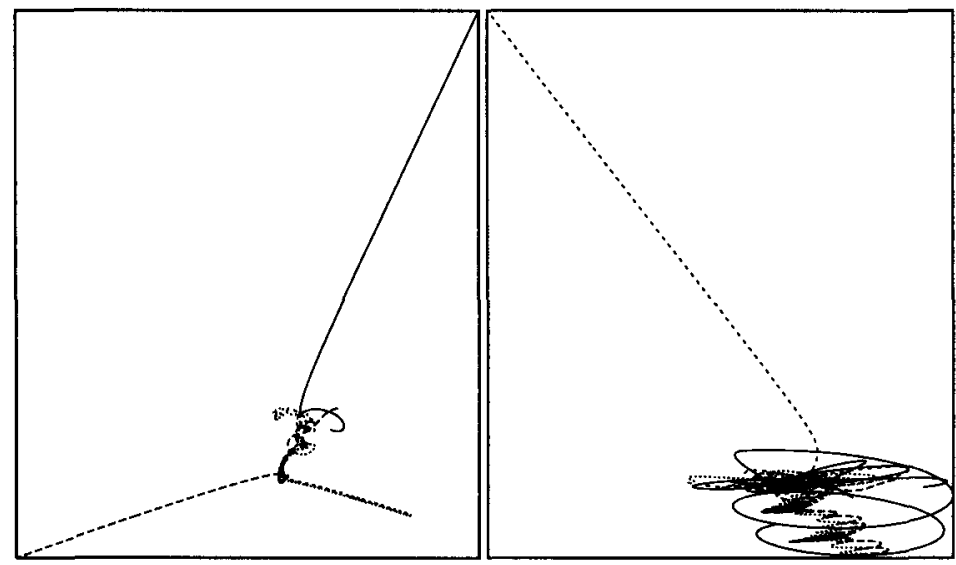

Figure 1. Examples of different final configurations of the systems. 
Case 5

Case6

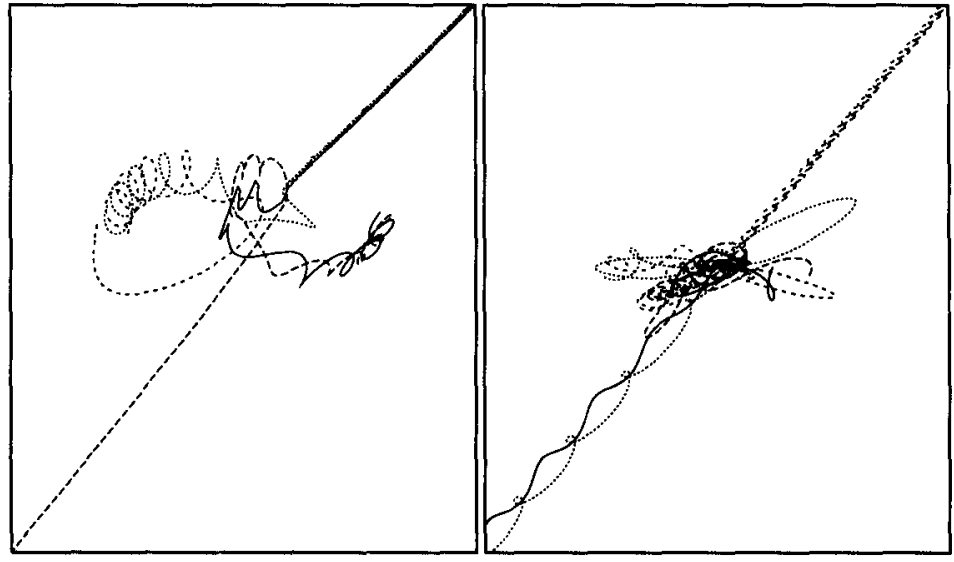

Case7

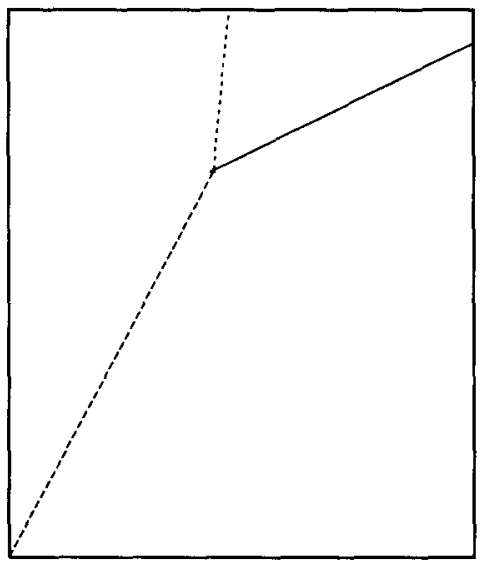

Figure 1. Continued. 
Due to the limitations of available computer time about half of the cases did not end up in any of the categories above. These are typically very loose hierachical systems whose evolution takes a very long time. In subsequent work (Heinämäki 1998) it has been demonstrated that the unclassifiable part of the sample does not bias the overall results.

Cases 2 and 4 represent one-sided ejections, while cases 3,5 and 6 represent two-sided ejections and case 7 three-sided ejections. On average, in a typical simulation about 30 percent of the cases ended up as one-sided ejections, 10 percent as two-sided ejections and a few percent as three-sided ejections.

These results can be applied to different black hole ejection processes, with different sample selection properties depending on the mode of visibility of the black holes in observations. An application to double radio sources (Saslaw et al. 1974, Valtonen 1999, Valtonen and Heinämäki 2000) is described in Heinämäki and Valtonen (2000).

\section{References}

Heinämäki, P. 1998, PhD Thesis, Univeristy of Turku

Heinämäki, P. and Valtonen, M. J. 2000, these proceedings

Peters, P. C. and Mathews, J. 1963, Phys. Rev., 131, 435

Saslaw, W. C., Valtonen, M. J. and Aarseth, S. J. 1974, ApJ, 190, 253

Valtonen, M. J., Mikkola, S., Heinämäki, P. and Valtonen H. 1994, ApJS, 95, 69

Valtonen, M. J. 1999, ApJ, 520, 97

Valtonen M. J. and Heinämäki, P. 2000, ApJ, 530. (To appear Feb 10, 2000 edition). 